\title{
Éditorial du dossier « Tests prénataux non invasifs »
}

\author{
Editorial for the Thematic File "Non-Invasive Prenatal Testing"
}

\author{
D. Sanlaville \\ (C) Lavoisier SAS 2016
}

Dans ce numéro de la Revue de médecine périnatale de mars 2016, le dossier thématique est consacré aux tests non invasifs en période prénatale. Depuis quelques années ces tests se développent et sont transférés en pratique clinique. En 2012, dans cette même revue (Vol. 4, numéro 2, juin 2012) un article sur le dépistage prénatal non invasif (DPNI) décrivait le principe et l'arrivée des tests non invasifs en diagnostic.

Dans ce dossier thématique seront abordées les applications des test non invasifs en période prénatale.

Ces tests ont pour principal avantage, outre le fait qu'ils ne sont pas invasifs, d'éliminer le risque de fausses couches suite au prélèvement. On peut distinguer des tests diagnostiques comme le diagnostic de sexe fœtal ou le génotypage rhésus et des tests de dépistage comme la recherche d'une trisomie 21 chez le fœetus qui est certainement le test non invasif le plus médiatisé.

En effet, de nombreux articles scientifiques ont validé l'intérêt et l'efficacité de ce test. À titre d'exemple, la valeur prédicative positive (VPP) du test combiné du premier trimestre est de $4 \%$ (donnée de l'Agence de la biomédecine) alors que la VPP du DPNI pour une population à risque de trisomie 21 fœtale est de $99 \%$. Par ailleurs, ce test est devenu disponible en France il y a environ deux ans et son coût ne cesse de baisser.

La Haute autorité de santé (HAS) a publié un communiqué de presse le 13 novembre 2015 intitulé « Trisomie 21 : de nouveaux tests appellent la révision de la stratégie de dépistage $»$. Une étude médico-économique (SAFE21 pilotée par le Pr Salomon, hôpital Necker) est en cours. L'HAS continue son travail et abordera dans un rapport la question de l'évaluation, des enjeux éthiques, économiques et organisationnels puis définira précisément la place de ces tests dans la stratégie de dépistage de la trisomie 21 en France.

\section{Sanlaville $(\bowtie)$}

Service de génétique, centre de biologie et de pathologie Est, 59 boulevard Pinel, F-69677 Bron cedex

e-mail : damien.sanlaville@chu-lyon.fr
Un article fera la synthèse sur les pratiques du DPNI dans plusieurs pays francophones. Il montrera la grande différence de pratique selon les pays.

Le terme DPNI sous-entend dépistage prénatal non invasif de la trisomie 21. Il s'agit toutefois d'un mauvais terme, car le test de dépistage combiné du premier trimestre est également un test non invasif. Dans ce dossier, le terme DPNI est dans la majorité des cas utilisé pour signifier le test de dépistage non invasif de la trisomie 21 sur ADN fœtal circulant. Ce terme devrait évoluer et les Anglosaxons parle plutôt de « cell-free DNA (cfDNA) based non-invasive prenatal testing ».

Outre la trisomie 21 , ces tests permettent de rechercher bien d'autres anomalies chromosomiques, dont des anomalies des gonosomes. Un article est consacré à ce sujet qui soulève également des problèmes éthiques.

En dehors des anomalies chromosomiques, ces tests peuvent également identifier des variations nucléotidiques dans un gène et il est maintenant possible de proposer un test non invasif pour des maladies géniques bien connues comme la mucoviscidose. Cette application sera abordée dans un des articles de ce numéro.

Face à ces avancées et ces nouvelles possibilités, il ne faut pas oublier le futur enfant à naître et les nombreuses questions éthiques qui se posent, dont la question d'un retour à l'eugénisme. Ainsi, il nous a paru important de consacrer un article à ces questions, de même qu'il était essentiel de connaître le point de vue des usagers et la perception des couples et des femmes sur le DPNI. Pour de nombreux usagers, il est difficile de comprendre pourquoi ce test n'est pas remboursé en France. Ainsi, un article sur les contraintes administratives menant au remboursement d'un test diagnostique a été réalisé.

En conclusion, nous rentrons dans une nouvelle ère avec des changements rapides concernant les tests en période prénatale. Au niveau cytogénétique, certains articles commencent à parler de caryotype fotal sur ADN fœtal circulant et nous devrons certainement adapter nos pratiques dans les mois à venir.

Pour des raisons éditoriales, nous avons dû scinder ce dossier en deux numéros. Vous trouverez donc la suite des articles dans le numéro de juin 2016. 